\title{
Amperometric Electrochemical Sensor with Multiple Working Electrodes to Determine Direction of Chemical Flow
}

\author{
Tomomi Makishita, Jin Miyasaka ${ }^{1}$ and Hiroshi Ishida* \\ Department of Mechanical Systems Engineering, Tokyo University of Agriculture and Technology, \\ 2-24-16 Nakacho, Koganei, Tokyo 184-8588, Japan \\ 'Department of Electrical and Electronic Engineering, Tokyo Institute of Technology, \\ 2-12-1 Ookayama, Meguro-ku, Tokyo 152-8552, Japan
}

(Received June 1, 2010; accepted August 2, 2010)

Key words: electrochemical sensor, amperometric sensor, shielding effect, disk electrode

A new method of detecting the flow direction of an aqueous solution of a chemical substance is proposed. The detection mechanism is based on the shielding effect that appears between multiple working electrodes of an amperometric electrochemical sensor. When the sensor is exposed to the flow of a chemical solution, a smaller response current is obtained at the downstream electrode than at the upstream electrode since a portion of the chemical substance is consumed at the upstream electrode. The response characteristics of amperometric sensors with dual, triple, and quadruple disk working electrodes were compared using an empirical model equation of the shielding effect with a constant shielding factor. The results showed that the quadruple disk electrodes are ideal in resolving the two-dimensional angle of the flow. A sensor probe with quadruple carbon disk electrodes was then fabricated. Its response characteristics were tested by placing the sensor in a laminar flow generated in a flow-through cell and in a turbulent flow generated in a water tank. The results of the experiments show that the fabricated sensor consistently responds to the local flow near the sensor surface. The potential applications of the proposed sensor include tracking of chemical plumes to find their sources and analyzing the flow around an object in fluid dynamics experiments.

\section{Introduction}

A variety of chemical sensors and biosensors have been developed to date for the detection of various chemical substances. ${ }^{(1)}$ Some sensors are commercially available and are already being used in various applications whereas others include newly proposed sensors based on novel sensing principles, which still require field tests. The basic function of these chemical sensors is to detect a specific class of chemical

${ }^{*}$ Corresponding author: e-mail: h_ishida@cc.tuat.ac.jp 
substances and possibly to quantify the concentration of the detected chemical. In some applications, however, this is not sufficient.

Water pollution monitoring, for example, generally involves the measurement of the concentration of a specific chemical substance at a fixed location over time. In some cases, however, the workers need to find the location of the chemical source in order to stop chemical leakage. When a chemical substance emanates from its source into a river or an ocean, a turbulent stream of water carries the released chemical and spreads it downstream. The molecular diffusion of a chemical substance into water is generally much slower than the background water flow. (2) Therefore, it is the turbulence of the water flow that mainly determines the structure of the chemical distribution. The streaks of the chemical released into the flow are tangled by a number of eddies in the turbulent flow, and form a randomly meandering chemical plume with a patchy internal structure. ${ }^{(2)}$ Underwater chemical-sensing robots are being developed to attain autonomous searches for chemical sources by tracking such chemical plumes. ${ }^{(3-5)}$ What has been highlighted from those works is the importance of detecting the direction of the chemical flow. Tracking the gradient of chemical concentration by simply comparing the signals from laterally aligned chemical sensors often fails in turbulent flow environments. Since a chemical plume is stretched by the flow in the flow direction, the gradient of the chemical concentration along the flow is extremely small. Moreover, the detected chemical concentration always contains large fluctuations due to the random nature of the turbulence. The direction of the flow carrying a chemical substance is thus a robuster cue in plume tracking since the source of the plume is always in the upstream direction from the point the chemical is detected.

A submersible sensor that can measure the direction of water flow is also a useful tool for experimental fluid dynamics. It is a common practice in investigating the flow around a certain structure to place a miniaturized model in a flume and measure the flow velocity around the model. Various technologies are available for flow measurement, ${ }^{(6)}$ but each one of them has both merits and demerits. Although accurate velocity measurements can be obtained using a laser Doppler velocimeter, the point of measurement must be accessible from an external laser light source. Therefore, it is difficult to measure the flow velocity along a complicated concave structure. It is also difficult to use an ultrasonic velocimeter near an object. Hot-wire and hot-film velocimeters enable highspeed measurement of local flow velocity. However, they cannot determine the flow direction. The heat taken away by the flow remains the same even when the flow starts to come from a completely opposite direction. Particle image velocimetry and particle tracking velocimetry provide rich information about the distribution of flow velocity vectors. However, it is again difficult to measure the flow velocity at locations very close to the object surface.

In this paper, we propose an amperometric electrochemical sensor that can detect the flow direction of a chemical plume. Here, we consider the electrochemical sensing of a single species of chemical substance using bare electrodes. However, amperometric electrochemical detection is widely used as a signal transduction mechanism in a variety of biosensors. ${ }^{(1)}$ The results described in this paper could be easily applied to the selective detection of a specific chemical substance using amperometric biosensors, e.g., 
enzyme electrodes. The proposed sensor is equipped with multiple working electrodes. When the sensor is immersed in a chemical plume or in a flow of a chemical solution, part of the chemical substance is consumed at the upstream electrodes. Therefore, only a decreased amount of the chemical reaches the downstream electrodes. Therefore, smaller response currents are obtained at the downstream electrodes than at the upstream electrodes. This phenomenon is known as the shielding effect. ${ }^{(7)}$ In ref. 8, we reported the results of our preliminary experiments using a sensor with dual working electrodes. We extend our work in this study to triple and quadruple working electrodes. The response characteristics of these sensors were first compared using the empirical model equation of the shielding effect, and the sensor with the quadruple working electrodes was chosen as a result. A sensor probe with quadruple carbon disk electrodes was then fabricated, and its response characteristics were tested in a laminar flow generated in a flow-through cell and in a turbulent flow generated in a water tank. In the following sections, the process of determining the best arrangement of the working electrodes and its experimental validation are described.

\section{Methods}

\subsection{Carbon disk electrodes}

The proposed sensor has multiple working electrodes for determining the direction of the chemical flow. To test the validity of the sensing principle, carbon disk electrodes shown in Fig. 1 were fabricated. Pencil leads with a diameter of $0.5 \mathrm{~mm}$ were first coated with a nonconductive thin polymer layer by applying a water-resistant spray. The leads were then encapsulated in a glass tube using epoxy resin. One end of the tube was polished flat so that the circular cross sections of the pencil leads were exposed to the solution. Carbon disk electrodes of the same size and shape were thus fabricated. They were connected to a custom-made potentiostat circuit and served as the working electrodes (WEs). Silver wire and a stainless-steel tube were used as the reference electrode (RE) and counterelectrode (CE), respectively.

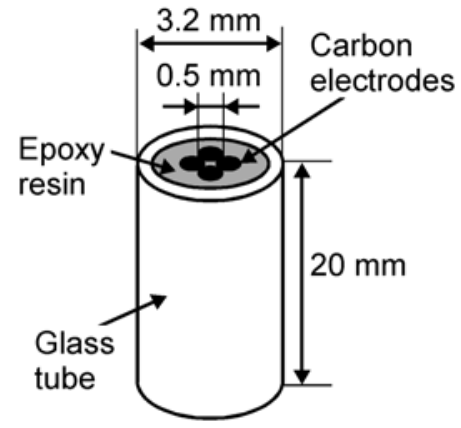

(a)

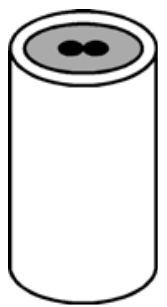

(b)

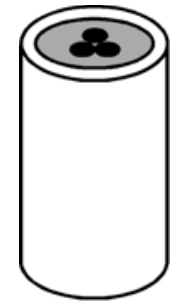

(c)

Fig. 1. Structures of (a) quadruple, (b) dual, and (c) triple disk electrodes. 
Ascorbic acid (vitamin C) was selected as the detection target since it can be easily detected with a carbon electrode. ${ }^{(9)}$ The aqueous solution of ascorbic acid of a constant concentration $(0.006 \mathrm{M})$ was used in the experiments. Sodium chloride $(0.5 \mathrm{M})$ was used as a support electrolyte mimicking the salinity in marine environments. All carbon electrodes were kept at a constant potential $(0.7 \mathrm{~V})$ with respect to the silver reference electrode. This potential is within the range in which the mass-transport-limited current for the oxidization of ascorbic acid is obtained.(9)

\subsection{Arrangement of working electrodes}

Before actually fabricating the carbon electrodes and testing them in ascorbic acid solution, the angular response characteristics of dual, triple, and quadruple working electrodes were investigated using an empirical model equation of the shielding effect. As mentioned earlier, the working electrodes placed downstream show smaller current responses than those placed upstream. The extent to which the current at a downstream electrode decreases depends on various parameters including the shape of the electrodes and the gap between them. The shielding effect between the ring and disk electrodes has been well characterized.(7) However, a theoretical equation that can be used to predict the amount of current decrease for multiple disk electrodes is not available. Instead, we used a simple model of the shielding effect with a constant shielding factor to predict the responses of the disk electrodes.

Suppose that the flow is uniform at least at the scale of the diameter of the sensor. This assumption is required to ensure that all working electrodes are exposed to the same flow. As shown in Fig. 2, the working electrodes are numbered sequentially in the counterclockwise direction when looking down at the electrode surface. The direction of the flow is defined as the counterclockwise angle from working electrode 1 (WE 1). In the following discussions, $I_{k}$ represents the current obtained from the $k$-th working electrode $(k=1,2,3,4) . I_{k 0}$ denotes the current from the $k$-th electrode when it is used independently without being shielded by the other electrodes. Ideally, $I_{k 0}$ is the same for all electrodes. However, it varies for different electrodes because of the variations in the size of the electrodes and their surface roughness. Therefore, the normalized response

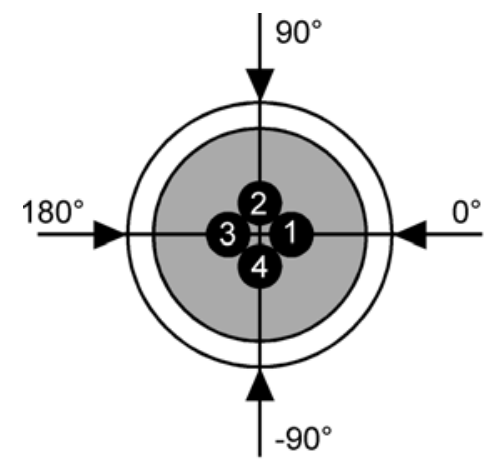

Fig. 2. Definition of flow direction with respect to disk electrodes. 
current $I_{k} / I_{k 0}$ is compared among different electrodes. The normalized current difference between the $j$-th and $k$-th electrodes, $\Delta I_{j k}$, is defined as

$$
\Delta I_{j k}=I_{j} / I_{j 0}-I_{k} / I_{k 0}
$$

and is used to determine the direction of the chemical flow.

Figure 3(a) shows the arrangement of the dual disk electrodes. The response current of an amperometric sensor is proportional to the area of the working electrode. In Fig. $3(\mathrm{a})$, area $\alpha$ of WE 2 is directly exposed to the incoming chemical solution. On the other hand, the solution that reaches area $\beta$ of WE 2 contains a smaller amount of the chemical substance because of the existence of WE 1 on the upstream side. Therefore, the current obtained from WE 2 is represented as

$$
I_{2}=\frac{\alpha}{\alpha+\beta} I_{20}+K \frac{\beta}{\alpha+\beta} I_{20}
$$

where $K$ is the shielding factor. $K$ represents the factor by which the response current is decreased owing to the shielding effect and is therefore always less than unity. $K$ varies for different arrangements of the electrodes. However, we assume, for simplicity, that $K$ is constant regardless of the shape of the electrodes and the gap between them. The normalized current difference between WEs 1 and 2 is then written as

$$
\Delta I_{12}=I_{1} / I_{10}-I_{2} / I_{20}=1-\frac{\alpha+K \beta}{\alpha+\beta}
$$

since $I_{1}$ is equal to $I_{10}$ in Fig. 3(a).

Figure 4 shows the angular variation of $\Delta I_{12}$ for the dual disk electrodes. For each direction, $\Delta I_{12}$ was obtained from eq. (3) by calculating the areas of $\alpha$ and $\beta$ in that specific flow direction. The shielding factor $K$ was determined to be 0.78 from the

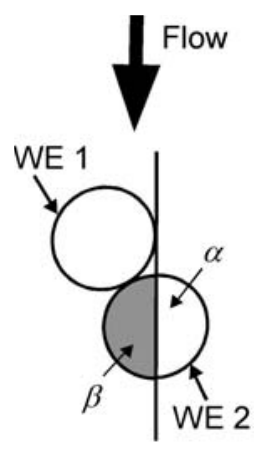

(a)

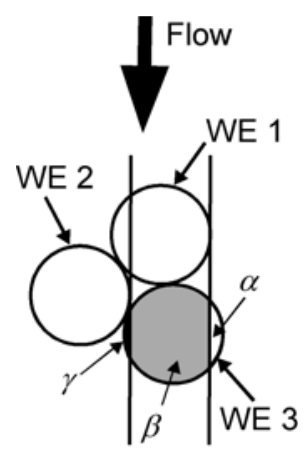

(b)

Fig. 3. Partial areas of electrodes influenced by shielding effect. (a) Dual disk electrodes. (b) Triple disk electrodes. 


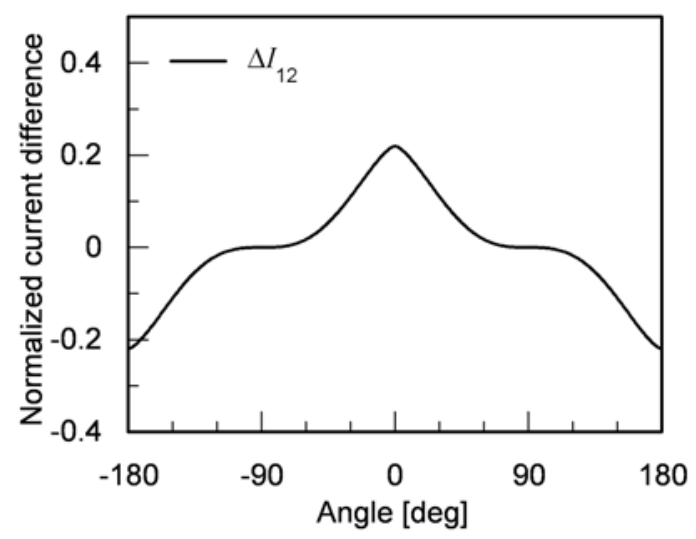

Fig. 4. Angular response characteristics of dual disk electrodes.

experiments using dual carbon disk electrodes exposed to a flow of $1.3 \mathrm{~cm} / \mathrm{s}$. This same value is also used in the following discussions. The angular response characteristics shown in Fig. 4 agree well with the experimental data reported in ref. 8. $\Delta I_{12}$ becomes positive in the flow direction of approximately $0^{\circ}$ and becomes negative in the flow direction of approximately $180^{\circ}$. Even though those two directions are easily discriminated, a problem arises when the flow comes from a $90^{\circ}$ or $-90^{\circ}$ direction. For example, $\Delta I_{12}$ stays almost the same for angles from 60 to $120^{\circ}$. Therefore, the direction of the flow in this range cannot be determined precisely. This result led us to try triple and quadruple disk electrodes.

For the triple disk electrodes, we need to consider the case shown in Fig. 3(b). Area $\alpha$ of WE 3 is directly exposed to the incoming flow of the chemical solution. The response current from area $\beta$ of WE 3 is affected by WE 1 exactly in the same way as in the case of the dual disk electrodes. However, care should be taken for the response current of area $\gamma$ of WE 3. The chemical solution that reaches area $\gamma$ has passed over WEs 1 and 2. We assume that the response current of WE 3 in this case is represented as

$$
I_{3}=\frac{\alpha}{\alpha+\beta+\gamma} I_{30}+K \frac{\beta}{\alpha+\beta+\gamma} I_{30}+K^{2} \frac{\gamma}{\alpha+\beta+\gamma} I_{30}=\frac{\alpha+K \beta+K^{2} \gamma}{\alpha+\beta+\gamma} I_{30} .
$$

The angular response characteristics calculated for the triple disk electrodes are shown in Fig. 5. There are still some angular regions in which the variation in the normalized current difference becomes small. For example, from 30 to $90^{\circ}$, the normalized current difference for all three pairs in the triple disk electrodes stays almost the same. Therefore, the small variation in the flow direction cannot be identified in this region.

Figures 6 and 7 show the angular response characteristics of the quadruple disk electrodes. The normalized current difference for adjacent electrode pairs is shown in Fig. 6. The normalized current difference for a diagonally aligned electrode pair is shown in Fig. 7. In contrast to the response characteristics of the dual and triple disk electrodes, 


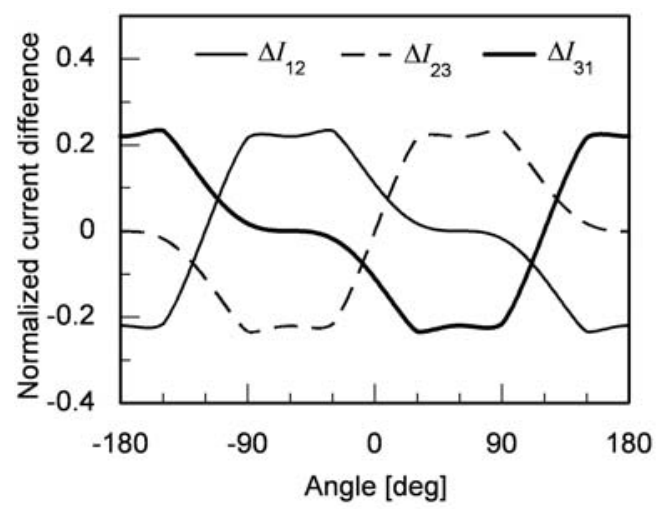

Fig. 5. Angular response characteristics of triple disk electrodes.

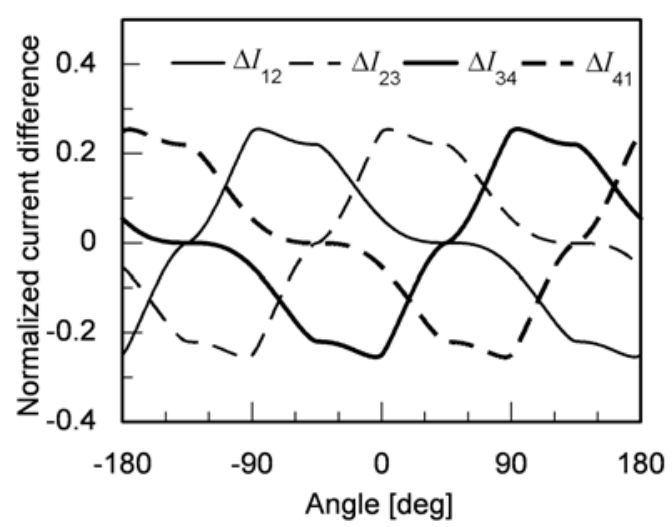

Fig. 6. Angular response characteristics of adjacent electrode pairs in quadruple disk electrodes.

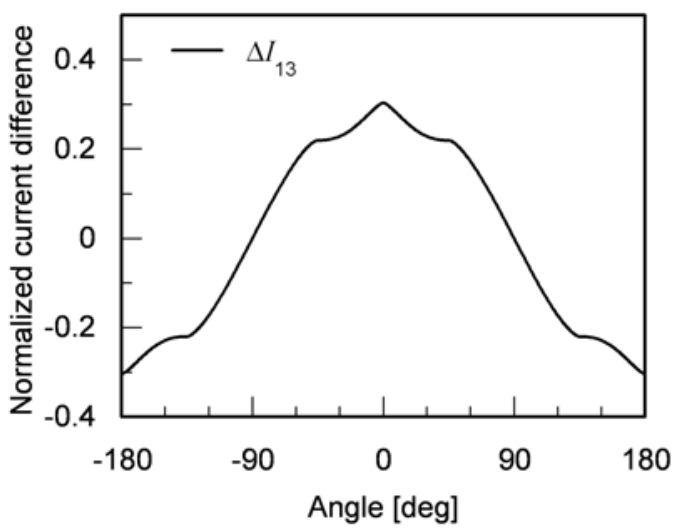

Fig. 7. Angular response characteristics of the diagonal electrode pair in quadruple disk electrodes. 
the quadruple disk electrodes show unique response patterns for the chemical flow from different directions. The two-dimensional direction of the incoming chemical flow can be unequivocally determined. Therefore, we decided to fabricate the quadruple disk electrodes as shown in Fig. 1(a) and test them in the experiments.

\section{Experimental Results and Discussion}

\subsection{Sensor response measurements in flow-through cell}

The fabricated quadruple disk electrodes were first tested in a one-dimensional laminar flow. For this purpose, the electrodes were placed in a flow-through cell as shown in Fig. 8. The cell consists of a Plexiglas block having a hole with an internal diameter of $4 \mathrm{~mm}$. Figure 9 shows the internal structure of the cell. Ascorbic acid solution $(0.006 \mathrm{M})$ was delivered through the hole using a peristaltic pump with different flow velocities $(0.93-9.3 \mathrm{~cm} / \mathrm{s})$. The stainless steel tube placed at the end of the hole served both as the drain electrodes and counterelectrode.

The response of an amperometric electrochemical sensor depends both on the concentration of the target chemical and on the velocity of the flow. Figure 10 shows
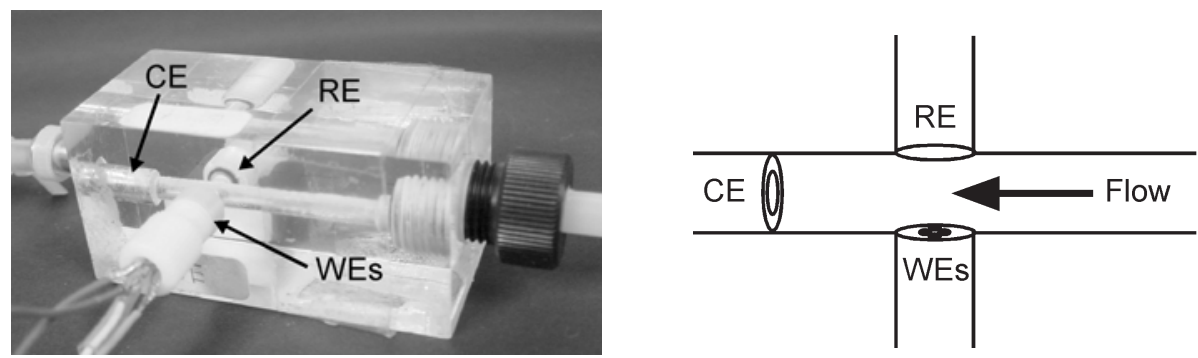

Fig. 8 (left). Flow-through cell for testing proposed sensor.

Fig. 9 (right). Schematic diagram of internal structure of flow-through cell.

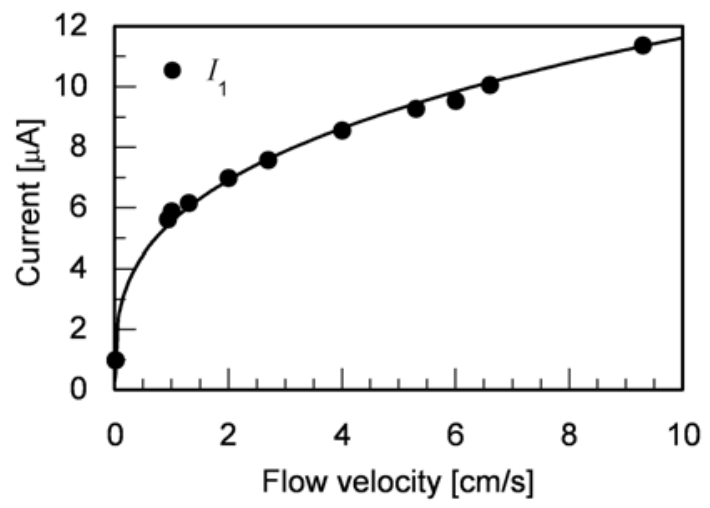

Fig. 10. Dependence of response current from WE 1 on flow velocity in flow-through cell. WE 1 was placed at the most upstream location. 
the response current at WE 1 when the flow direction was set to $0^{\circ}$. In this case, WE 1 was placed at the most upstream location. Theoretical analysis of the electrode exposed to a laminar flow in a pipe has shown that the response current is proportional to the $1 / 3$ power of the velocity. ${ }^{(10)}$ The current measurements agreed well with the theoretical values. Figures 11 and 12 show the normalized current difference for adjacent electrode pairs and a diagonal pair, respectively. The flow velocity was set to $5.3 \mathrm{~cm} / \mathrm{s}$. The quadruple disk electrodes were exposed to the flow in various directions by changing the orientation of the electrodes in the cell. The obtained response curves are somewhat distorted since slight misalignments are inevitable for the handmade electrodes and the flow-through cell. Nonetheless, the response curves are quite similar to those predicted by the empirical model equation. The normalized current difference of at least one sensor pair always changes with the angle of the flow. Therefore, the flow direction can

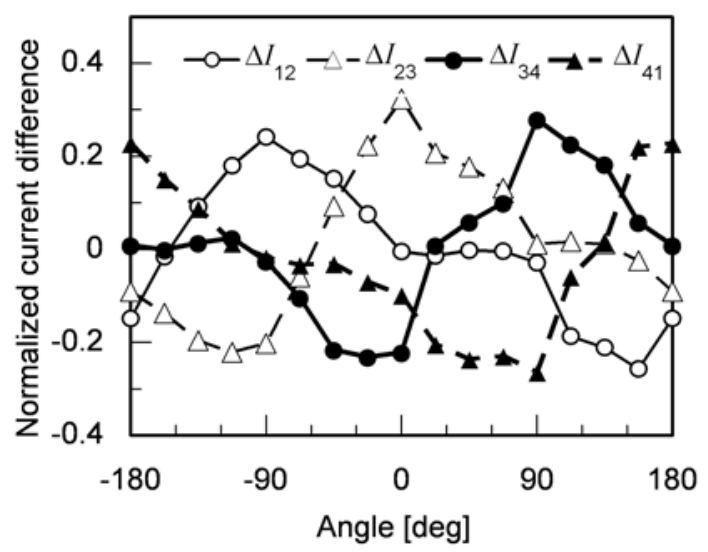

Fig. 11. Normalized current difference measured for adjacent electrode pairs in flow-through cell.

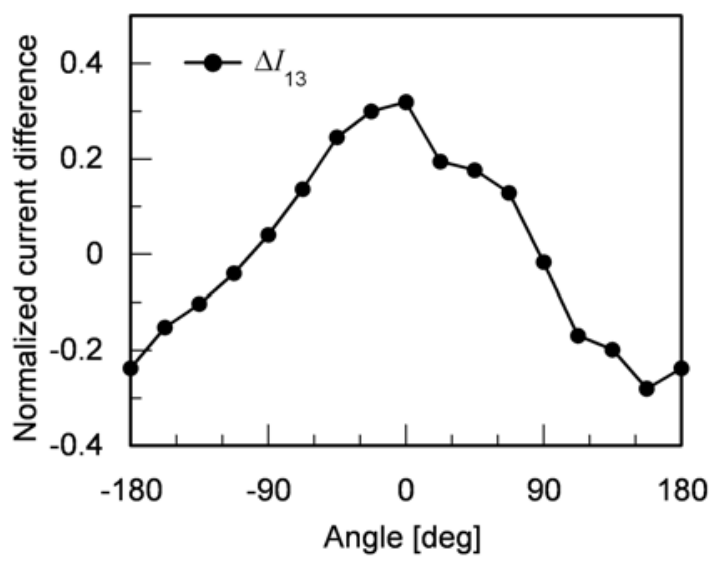

Fig. 12. Normalized current difference measured for diagonal electrode pair in flow-through cell. 
be easily identified. The validity of the empirical model and the sensing principle of the quadruple disk electrodes are thus shown for the laminar flow.

In ref. 4, results of chemical plume tracking experiments using an autonomous underwater robot were presented. The task given to the robot was to track a chemical plume in the upstream direction to find the origin of the chemical plume. The experiments were conducted near the shore of San Clemente Island, CA, and the velocity of the ocean flow observed during the experiments was in the range of 2 to $12 \mathrm{~cm} / \mathrm{s} .{ }^{(4)}$ We tested our sensor in the flow-trough cell over a similar velocity range (0.93-9.3 $\mathrm{cm} / \mathrm{s}$ ), and the sensor worked well for all flow velocities tested in the experiments. The shielding factor $K$ was almost constant over this velocity range.

The drawback of the proposed sensor is that the magnitude of the response currents depends both on the concentration of the chemical substance and on the velocity of the flow. As shown in Fig. 10, the response current increases with the flow velocity under a constant chemical concentration. If the flow velocity stays the same, the sensor response current increases with the chemical concentration. If both the concentration and flow velocity are unknown, these cannot be determined from the response current. However, if the concentration is known, the velocity can be estimated from the response current, and vice versa. Moreover, the direction of the flow can always be determined even if both the concentration and the velocity are unknown.

\subsection{Sensor response measurements in water tank}

As a next step after the experiments described in the previous section, the response characteristics of the quadruple disk electrodes immersed in an open channel flow were investigated. The experimental setup is shown in Fig. 13. A Plexiglas water tank was filled with $0.006 \mathrm{M}$ ascorbic acid solution to a depth of $3 \mathrm{~cm}$. A circulating flow was generated by placing a submersible motor with propeller fans (Submarine Motor, Tamiya Inc.) at the bottom of the water tank. The round corners and the central island of the

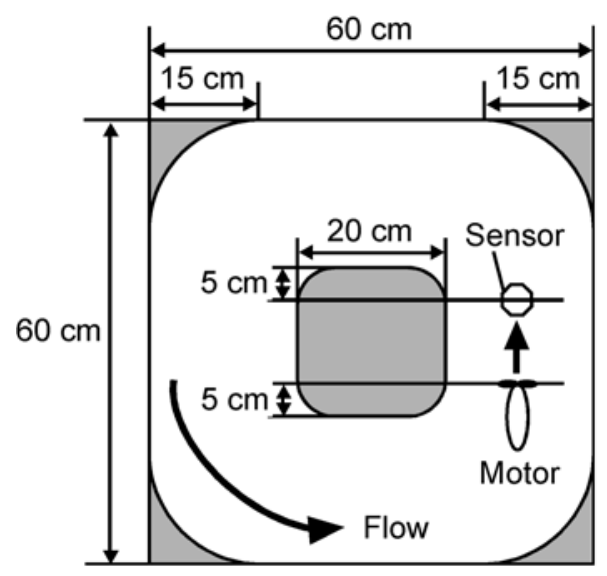

Fig. 13. Schematic diagram of the water tank (top view). 
tank were made of Styrofoam. The quadruple disk electrodes were placed $10 \mathrm{~cm}$ from the motor and exposed to the turbulent flow from the propeller fans. The flow velocity measured by introducing tracer particles on the water surface was $20 \mathrm{~cm} / \mathrm{s}$ at the place of the sensor.

In the experimental setup, the electrodes were exposed to the turbulent flow containing three-dimensional random fluctuations. If the flow velocity vector has a large component in the direction orthogonal to the surface of the electrodes, the shielding effect does not take place, as shown in Fig. 3. To suppress the three-dimensional motion of the ascorbic acid solution near the sensor surface, the sensor was embedded in a Plexiglas plate with an octagonal shape, as shown in Fig. 14. The surfaces of the disk electrodes were aligned with the surface of the octagonal plate to make a single flat surface.

We first placed the sensor as shown in Fig. 15(a) to make the octagonal plate and the sensor surface parallel to the surface of the water. Figure 16 shows the response currents measured when WE 1 was placed on the upstream side and WE 3 was on the downstream side. The result is misleading since WE 1 on the upstream side showed a smaller current response. When the sensor was rotated by $180^{\circ}$ to position WE 3 on the upstream side, the current response from WE 3 became smaller than the current response from WE 1.

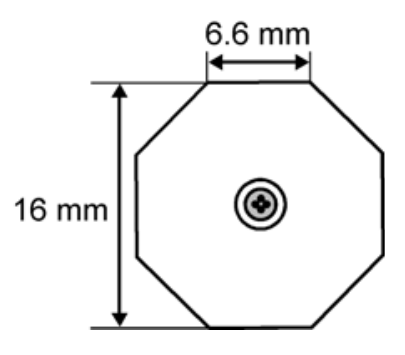

(a)

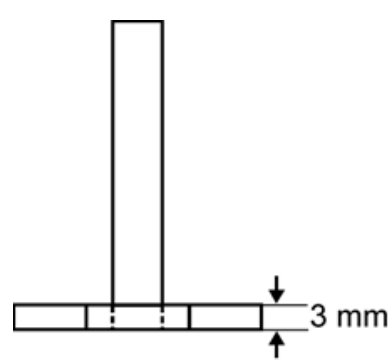

(b)

Fig. 14. Sensor with quadruple disk electrodes embedded in octagonal plate. (a) Bottom view. (b) Side view.

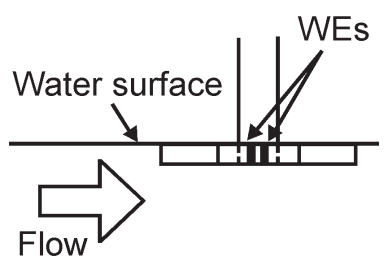

(a)

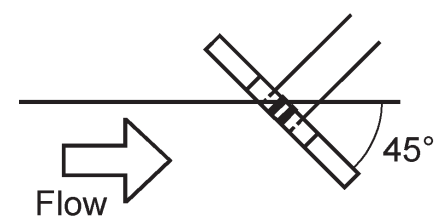

(b)

Fig. 15. Arrangement of sensor with respect to surface of water in tank. (a) Horizontal arrangement. (b) Tilted arrangement. 


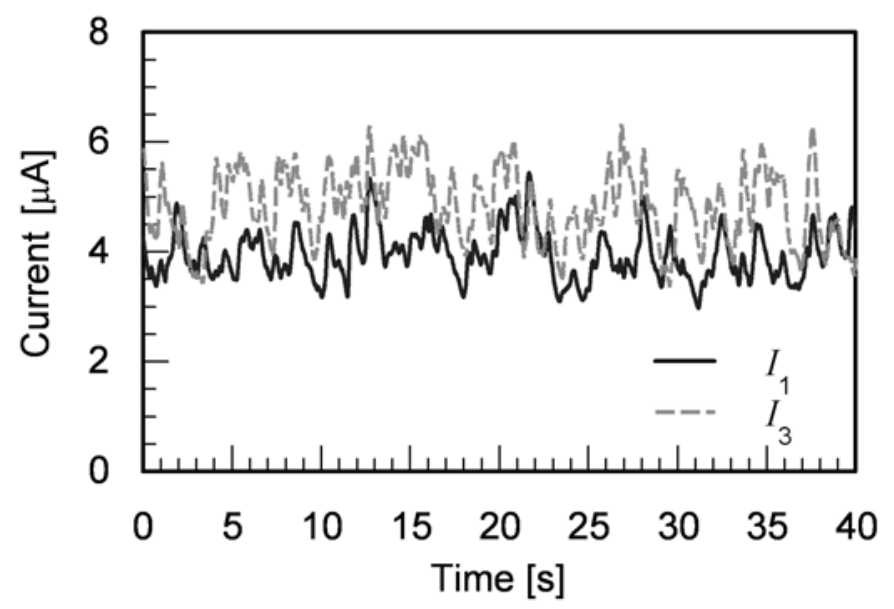

Fig. 16. Response currents from WEs 1 and 3 for sensor with octagonal plate placed horizontally.

In the flow-through cell, the current responses corresponding to the flow direction were successfully obtained. However, conflicting results were obtained from the experiments using the water tank.

After trying several different setups, we finally found a reasonable explanation for the conflicting results. Figure 17 shows the response currents from WEs 1 and 3 when the sensor was tilted, as shown in Fig. 15(b). In this case, WE 1 was placed on the upstream side, and WE 3 was placed on the downstream side. A larger current was obtained from WE 1 , which correctly shows the presence of the flow in the direction from WE 1 to WE 3. These results are clearly explained by considering the flow field around the octagonal plate and sensor.

The results of the computational fluid dynamics (CFD) simulations of the flow field around the sensor are shown in Fig. 18. A commercial software package, ANSYS FLUENT, was used for the simulation. A computational domain of $10 \mathrm{~cm}$ width, 10 $\mathrm{cm}$ length, and $3 \mathrm{~cm}$ depth was prepared. The upper face of the computational domain was assumed to be the water surface, and the model of the octagonal plate was placed hanging from the water surface. The lower face of the computational domain was assumed to be the bottom of the water tank. The water flow at a constant velocity $(20 \mathrm{~cm} / \mathrm{s})$ was introduced into this computational domain, and a uniform pressure was assumed at the downstream end of the domain. The nonslip boundary condition was assumed on the surface of the octagonal plate and at the bottom of the water tank. Owing to the viscosity of water, the flow velocity on the surface of an object is always zero. The symmetric boundary condition was assumed at the side walls of the computational domain and the water surface to let the water slip at these locations. This condition is required to simulate the flow in a large water tank rather than to simulate the flow in a closed channel with the size of the computational domain. The number of 


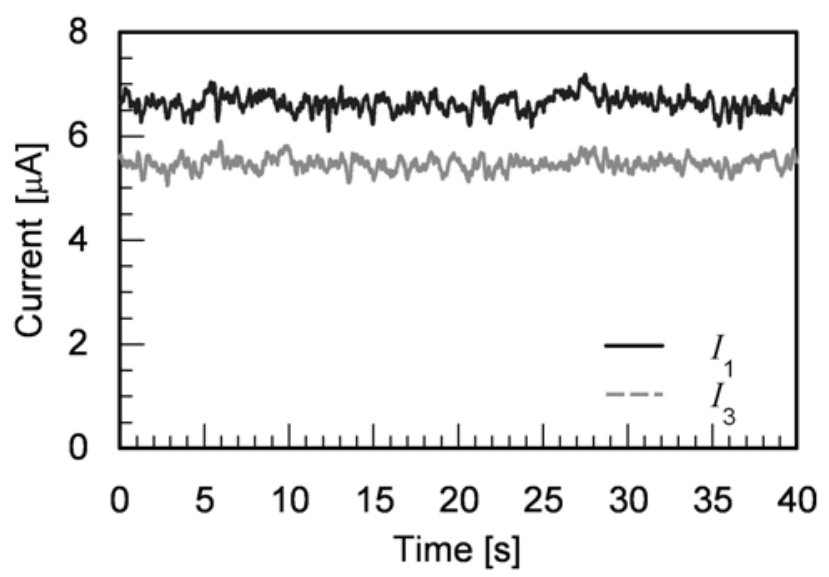

Fig. 17. Response currents from WEs 1 and 3 for tilted sensor.

grid points in the computational domain was 230,000, and the flow fields shown in Fig. 18 were obtained by assuming a steady-state flow. When the octagonal plate is placed horizontally, as shown in Fig. 18(a), the water flow separates at the upstream edge of the plate and starts to swirl. The quadruple disk electrodes of the sensor were placed at the center of the octagonal plate and were therefore exposed to the backflow in the swirl. This is the reason for the larger response current at WE 3. When the octagonal plate is tilted, as shown in Fig. 18(b), the sensor is exposed to the forward flow. Therefore, the larger response current was obtained from WE 1.

The results of the CFD simulations were further supported by some other experiments. In the simulations, the flow separation did not occur when the flow velocity was reduced to $10 \mathrm{~cm} / \mathrm{s}$. This result was experimentally confirmed by measuring the response currents at reduced flow velocities. In theory, the flow separation does not occur on a smooth surface. Therefore, we tested the rounded plate shown in Fig. 19. The result of the CFD simulation for this rounded plate is shown in Fig. 20. In the simulation, the water flow smoothly follows along the surface of the rounded plate even when the flow velocity is $20 \mathrm{~cm} / \mathrm{s}$. Therefore, no backflow is generated, as shown in Fig. 20. A clay model of the rounded plate was fabricated, and was placed together with the quadruple disk electrodes, as shown in Fig. 21. The response currents of WEs 1 and 3 are shown in Fig. 22. The measured response currents were smaller than those in Figs. 16 and 17 because of the repeated use of the same carbon electrodes. It is known that the response of a carbon electrode to ascorbic acid deteriorates over time. ${ }^{(9)}$ Nevertheless, a larger response current was correctly obtained from WE 1, which was placed on the upstream side. The flow separation was successfully suppressed by the rounded plate.

The experimental results shown in Figs. 16 and 17 show that the response currents reflect the direction of the local flow at the electrodes. In order to correctly determine the direction of the background bulk flow, the sensor must be embedded in a round 

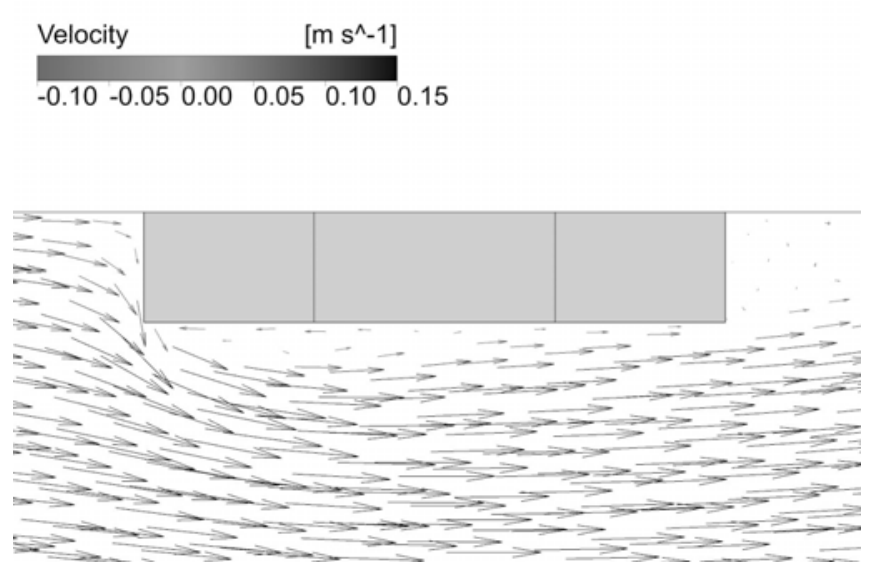

(a)
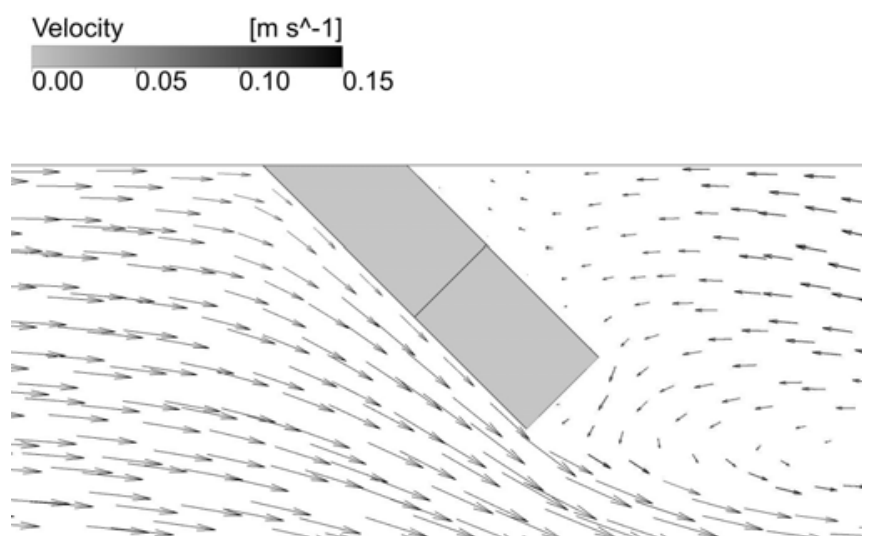

(b)

Fig. 18. Results of CFD simulation. (a) Flow around horizontally placed octagonal plate. (b) Flow around obliquely placed octagonal plate.

object as shown in Fig. 19. The shape of the object must be streamlined to avoid the flow separation. The streamlined shape also helps in minimizing the disturbances that the sensor introduces into the flow. The sensor embedded in a larger object has a lower spatial resolution in the flow direction measurement and also introduces larger disturbances into the flow. For tracking a flow of a chemical substance in the sea over 


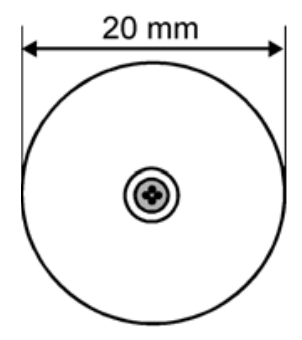

(a)

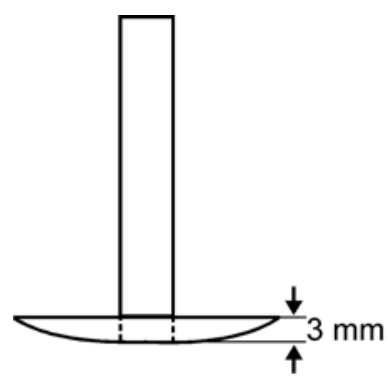

(b)

Fig. 19. Sensor with rounded plate. (a) Bottom view. (b) Side view.

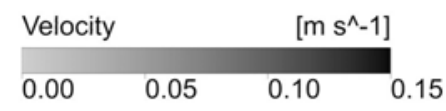

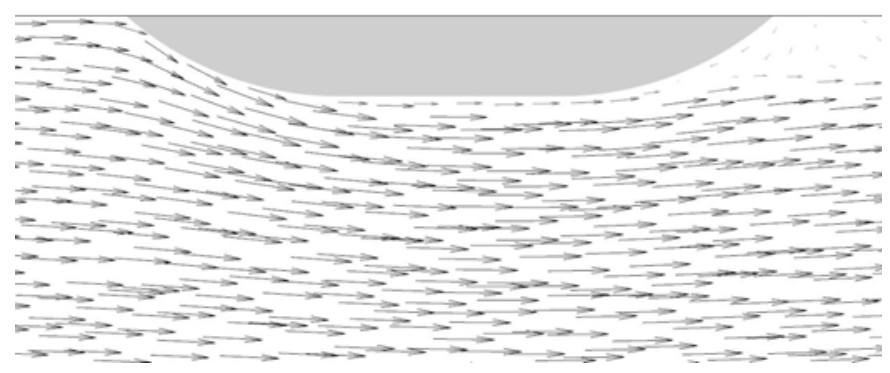

Fig. 20. Result of CFD simulation for flow around rounded plate.

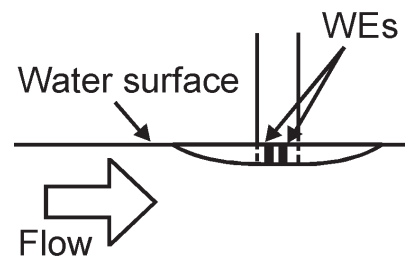

Fig. 21. Arrangement of rounded plate with respect to water surface.

several meters, disturbances introduced by the round plate of $20 \mathrm{~mm}$ diameter would be negligible. In the experiments shown in Fig. 13, the sensor was directly exposed to the highly fluctuated flow generated by the propeller fans. For measuring the direction of a smaller-scale flow with less turbulent intensity, a smaller plate would be preferable. 


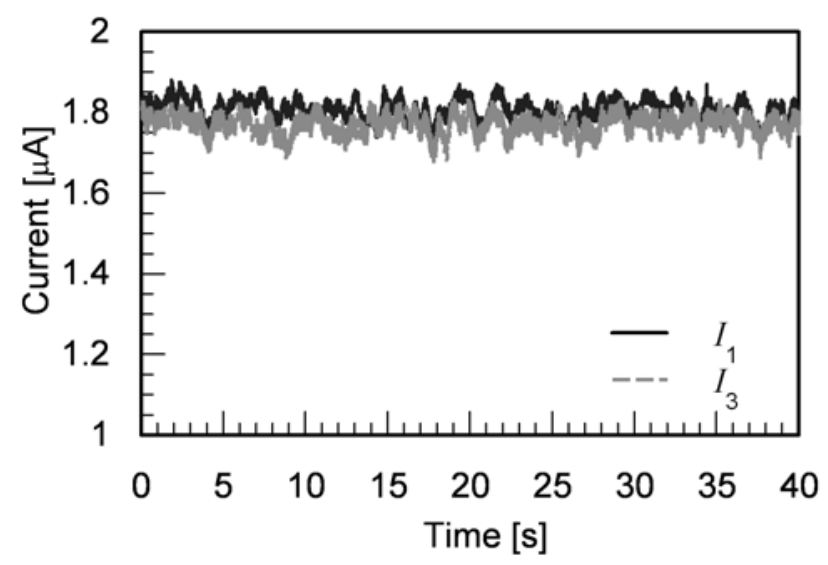

Fig. 22. Response currents from WEs 1 and 3 for sensor embedded in rounded plate.

The major limitation of the proposed sensor is its weak selectivity. For tracking a flow of a pollutant to find its source, the sensor must be capable of selectively detecting the target pollutant in the presence of interfering chemical substances. It is expected that such selective chemical detection will be accomplished by modifying the electrodes with an enzyme. ${ }^{(1)}$ The easiest way to modify the electrodes is to apply a layer of gel containing an enzyme on each electrode. In this case, however, the depletion of the target chemical substance owing to the electrochemical reaction occurs only in the thick enzyme layer, and the shielding effect will not appear. On the other hand, it is known that the depletion layer extends into the solution if the enzyme is immobilized at the electrode surface. ${ }^{(1,11)}$ In theory, electrodes with such a thin enzyme layer (a monolayer in most cases) can be used for the proposed sensor. In reality, however, the product of the electrode reaction may deteriorate the performance of the sensor as we observed in the smaller response current in Fig. 22. Although further work is needed to realize an enzymatic chemical flow direction sensor, it will surely become an interesting extension of this work. The use of enzymes will also enable the detection of chemical substances that are less reactive than ascorbic acid. The enzyme sensor will offer a wider selection of target chemical substances and will therefore have wider application areas.

\section{Conclusions}

We proposed the use of an amperometric electrochemical sensor equipped with multiple disk working electrodes to detect the direction of the chemical flow. The angular response characteristics of the dual, triple, and quadruple disk electrodes were first examined using a simple empirical model of the shielding effect. It was found that the quadruple disk electrodes were ideal in resolving the direction of the chemical flow. The quadruple carbon disk electrodes were then fabricated, and their response 
characteristics were examined. The results of the experiments and CFD simulations showed that the fabricated sensor responded consistently to the direction of the chemical flow near the sensor surface. When the sensor was attached to the octagonal plate and placed horizontally on the surface of the water flow, the response currents of the quadruple disk electrodes clearly showed the presence of the backflow near the sensor surface. These response characteristics of the sensors are favorable for fluid dynamics experiments. The flow field around the surface of an object can be measured simply by embedding multiple sensors in the object and dissolving a small amount of ascorbic acid in water. For plume tracking applications, the sensor needs to be mounted on a rounded surface to avoid obtaining the misleading responses. In order to make the proposed sensor work properly, there needs to be a chemical substance in the flow that can be easily reduced or oxidized at the carbon disk electrodes. The weak selectivity of the sensor is also problematic for the field use. Considering these limitations, the most immediate application of the proposed sensor would be in experimental fluid dynamics. To measure the flow distribution around an object, we can prepare ascorbic acid solution of a constant concentration and let it flow in a flume facility for hydrodynamic experiments. To use enzyme-modified electrodes would be a promising way to attain selective chemical detection in the field use. Future work will be addressed to develop an enzymatic chemical flow direction sensor.

\section{Acknowledgements}

We wish to gratefully acknowledge the helpful suggestions and comments by Dr. Jiri Janata of the School of Chemistry and Biochemistry, Georgia Institute of Technology and by Dr. Toyosaka Moriizumi, Emeritus Professor of Tokyo Institute of Technology. We also thank two anonymous reviewers for their valuable suggestions in revising the manuscript. Part of this work was supported by a Grant-in-Aid for Challenging Exploratory Research (No. 21650034), Japan Society for the Promotion of Science.

\section{References}

1 J. Janata: Principles of Chemical Sensors, Second Edition (Springer, New York, 2009).

2 P. J. W. Roberts and D. R. Webster: Turbulent Diffusion, in Environmental Fluid Mechanics, eds. H. Shen, A. Cheng, K.-H. Wang, M. H. Teng, and C. Liu (ASCE Press, Virginia, 2002) pp. $7-45$.

3 F. W. Grasso and J. Atema: Environ. Fluid. Mech. 2 (2002) 95.

4 W. Li, A. Farrell, S. Pang and R. M. Arrieta: IEEE Trans. Robot. 22 (2006) 292.

5 M. Ohashi, Y. Minagawa, Y. Myoren and H. Ishida: Proc. 7th Int. Conf. on Machine Learning and Applications (2008) pp. 482-488.

6 C. Tropea, A. L. Yarin and J. F. Foss (eds.): Handbook of Experimental Fluid Mechanics (Springer, Heidelberg, 2007).

7 A. L. Bard and L. R. Faulkner: Electrochemical Methods (John Wiley \& Sons, New York, 1980).

8 T. Nakamura, H. Ishida and T. Moriizumi: Tech. Dig. 18th Sensor Symp. (2001) pp. 439-442.

9 T. Kikas, H. Ishida and J. Janata: Anal. Chem. 74 (2002) 3605. 
10 V. G. Levich: Physicochemical Hydrodynamics (Prentice-Hall, Englewood Cliffs, NJ, 1962) p. 114.

11 J. J. Gooding, E. A. H. Hall and D. B. Hibbert: Electroanalysis 10 (1998) 1130.

\section{About the Authors}

Tomomi Makishita received her B.E. degree in Mechanical Systems Engineering from Tokyo University of Agriculture and Technology, Tokyo, Japan, in 2010. She is currently pursuing her M.E. degree in Mechanical Systems Engineering. Her research interest is in biomimetic robots with chemical sensing capabilities.

Jin Miyasaka received his B.E. degree in Electrical and Electronic Engineering from Tokyo Institute of Technology, Tokyo, Japan, in 2004. In 2006, he received the M.E. degree in Physical Electronics also from Tokyo Institute of Technology.

Hiroshi Ishida was born in Morgantown, WV, in 1970. He received his M.E. and Ph.D. degrees in Electrical and Electronic Engineering from Tokyo Institute of Technology, Tokyo, Japan, in 1994 and 1997, respectively. From 1997 to 2004, he was a Research Associate at Tokyo Institute of Technology. From 1998 to 2000, he visited the School of Chemistry and Biochemistry, Georgia Institute of Technology, Atlanta, GA, as a Postdoctoral Fellow. In 2004, he joined the Department of Mechanical Systems Engineering at Tokyo University of Agriculture and Technology, Tokyo, Japan, where he is currently an Associate Professor. In 2007, he was a visiting researcher in the AASS Research Centre, Örebro University, Sweden. His research interests are in biomimetic electronics with emphasis on chemical sensors and their applications in robotics. 